\title{
Crossover between Anti- and Pro-Oxidant Activities of Graphene Quantum Dots in the Absence or Presence of Light
}

Yu Chong, ${ }^{\dagger \dagger}$ Cuicui Ge,,${ }^{\dagger, \dagger}$ Ge Fang,${ }^{\dagger}$ Xin Tian, ${ }^{\dagger}$ Xiaochuan Ma,${ }^{\dagger}$ Tao Wen, ${ }^{\dagger}$ Wayne G. Wamer, ${ }^{\ddagger, 1}$ Chunying Chen, ${ }^{*}, \dot{ }{ }^{\perp}$ Zhifang Chai $,{ }^{\dagger},{ }^{\prime}$ and Jun-Jie Yin $* *$

${ }^{\dagger}$ School for Radiological and Interdisciplinary Sciences (RAD-X), Collaborative Innovation Center of Radiation Medicine of Jiangsu Higher Education Institutions, Soochow University, Suzhou 215123, China

Division of Bioanalytical Chemistry and Division of Analytical Chemistry, Office of Regulatory Science, Center for Food Safety and Applied Nutrition, U.S. Food and Drug Administration, College Park, Maryland 20740, United States

${ }^{\perp}$ Key Laboratory for Biomedical Effects of Nanomaterials and Nanosafety, National Center for Nanoscience and Technology of China and Institute of High Energy Physics, Chinese Academy of Sciences, Beijing 100190, China

*Corresponding authors. Email: chenchy@ nanoctr.cn; junjie.yin@ fda.hhs.gov; ccge@ suda.edu.cn

${ }^{1}$ Dedicated to the memory of Wayne G. Wamer 
(a)

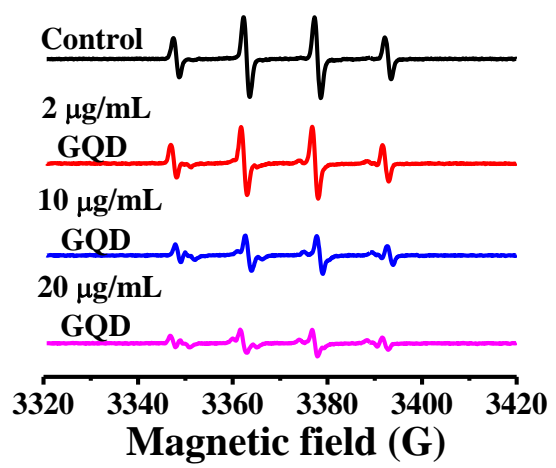

(b)

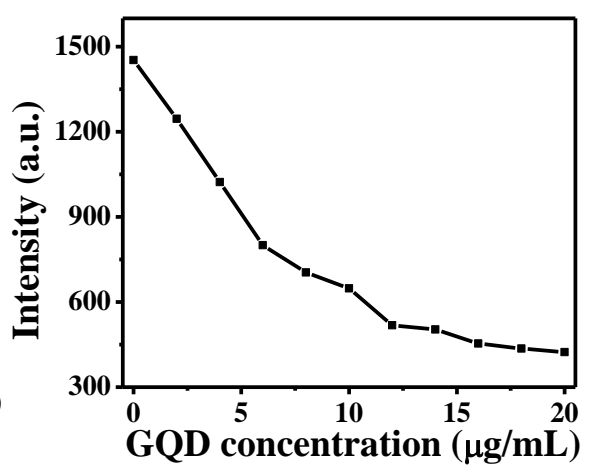

Figure S1. GQD as scavengers of hydroxyl radicals produced by irradiating $100 \mu \mathrm{g} / \mathrm{mL} \mathrm{TiO}_{2}$ with 340 nm UV light. (a) ESR spectra of the DMPO/ $\bullet \mathrm{OH}$ adduct obtained from samples containing $50 \mathrm{mM}$ DMPO, $100 \mu \mathrm{g} / \mathrm{mL} \mathrm{TiO}_{2}$ and different concentrations of GQD. (b) Effect of GQD concentration on their hydroxyl scavenging activity. All data were recorded after $1 \mathrm{~min}$ of irradiation at room temperature. 
(a)

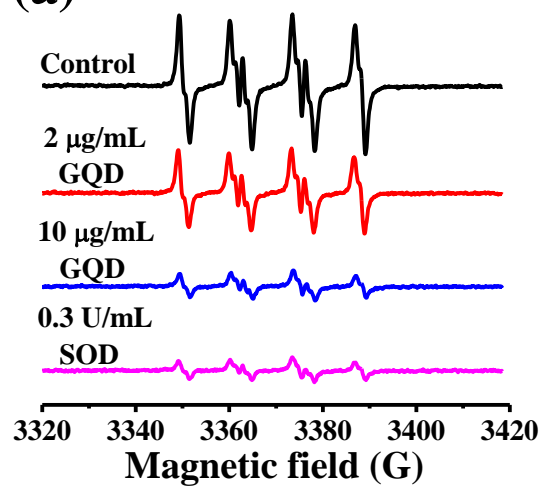

(b)

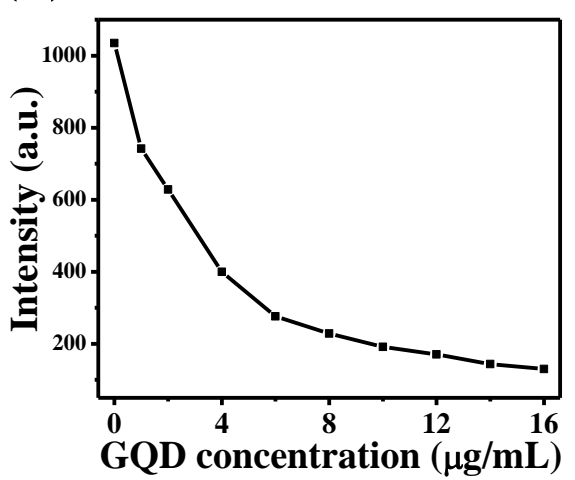

Figure S2. GQD as scavengers of superoxide radical anions generated by Xan/XOD. (a) ESR spectra of the BMPO/.OOH adduct obtained from samples containing $25 \mathrm{mM}$ BMPO, $1 \mathrm{mM}$ Xan, $0.1 \mathrm{U} / \mathrm{mL}$ XOD, $0.05 \mathrm{mM}$ DTPA and different concentrations of GQD. (b) Effect of GQD concentration on their superoxide scavenging activity. All data were recorded after $5 \mathrm{~min}$ of incubation at room temperature. 
(a)

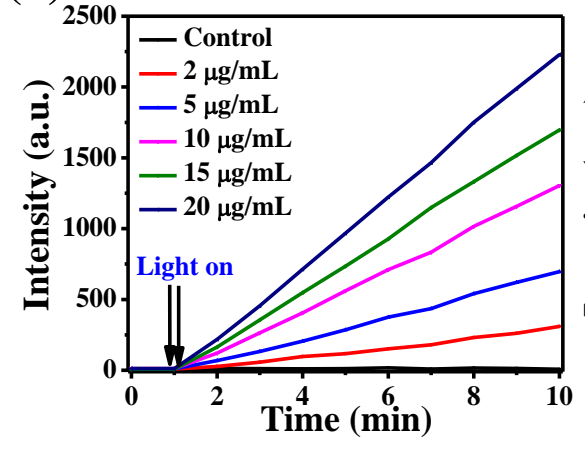

(b)

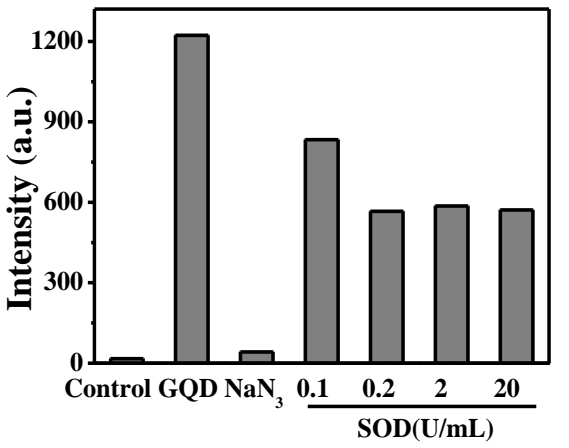

Figure S3. Generation of singlet oxygen. (a) Irradiation time and GQD concentration dependence of the ESR signal for the formation of 4-oxo-TEMP $/{ }^{1} \mathrm{O}_{2}$ adducts. Samples contained $10 \mathrm{mM}$ 4-oxo-TEMP and GQD varying in concentration from 2-20 $\mu \mathrm{g} / \mathrm{mL}$. (b) The effect of SOD and $\mathrm{NaN}_{3}$ on the 4-oxo-TEMP $/{ }^{1} \mathrm{O}_{2}$ adducts generated from irradiated GQD. Samples contained $10 \mathrm{mM}$ 4-oxo-TEMP, $20 \mu \mathrm{g} / \mathrm{mL} \mathrm{GQD} \mathrm{and} \mathrm{SOD} \mathrm{or} \mathrm{NaN}_{3}$. 


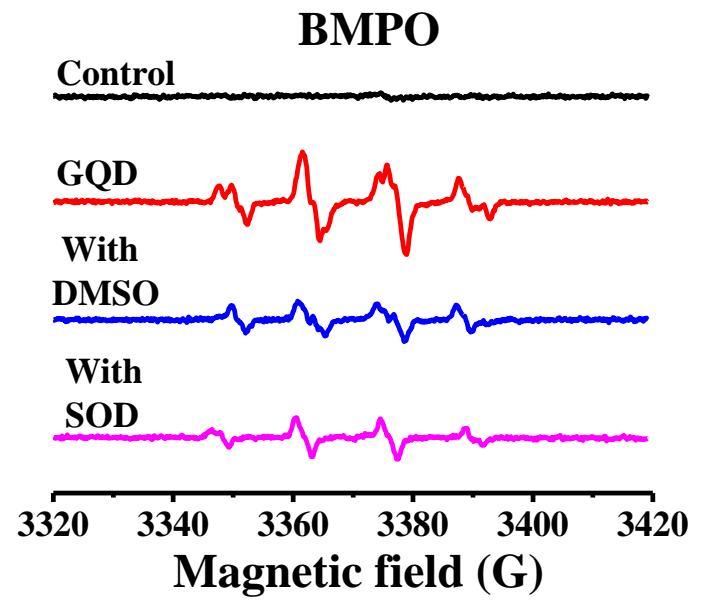

Figure S4. Effect of SOD and DMSO on the ESR signal of BMPO generated from $100 \mu \mathrm{g} / \mathrm{mL}$ GQD when exposed to light. 


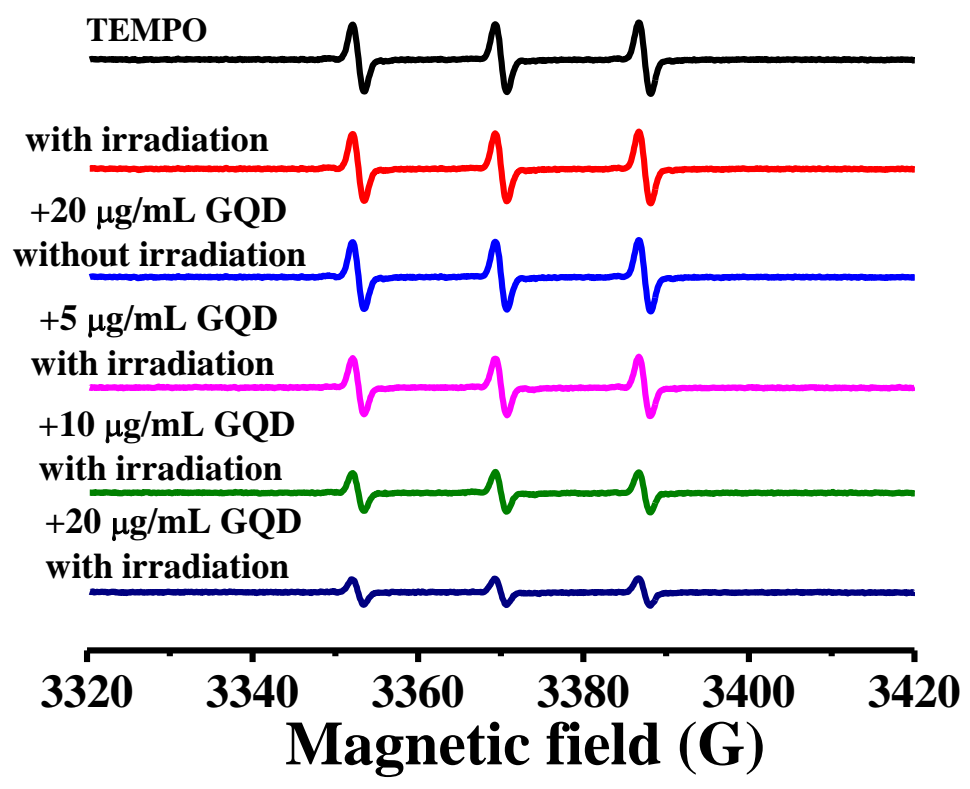

Figure S5. Reduction of TEMPO by photoexcited GQD. Spectra were obtained after irradiation for 5 min. ESR spectra obtained from samples containing $20 \mu \mathrm{M}$ TEMPO, $10 \mathrm{mM}$ PBS buffer (pH 7.27) and different concentrations of GQD. 
(a)

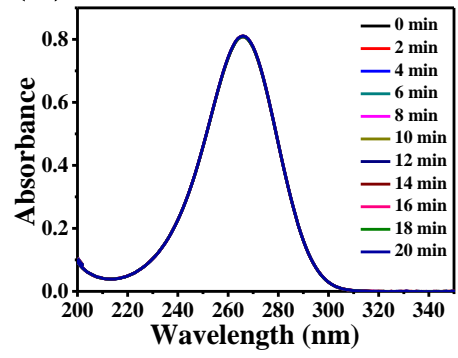

(b)

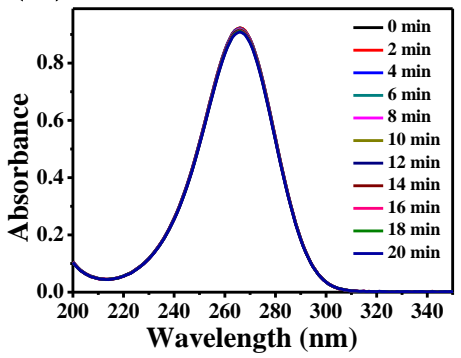

(c)

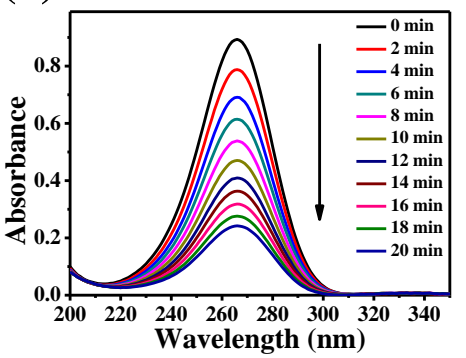

Figure S6. Evolution of UV-vis absorption spectra for ascorbic acid $(100 \mu \mathrm{M})$ in the presence of light (a), $10 \mu \mathrm{g} / \mathrm{mL}$ GQD (b) and irradiated GQD (c). 

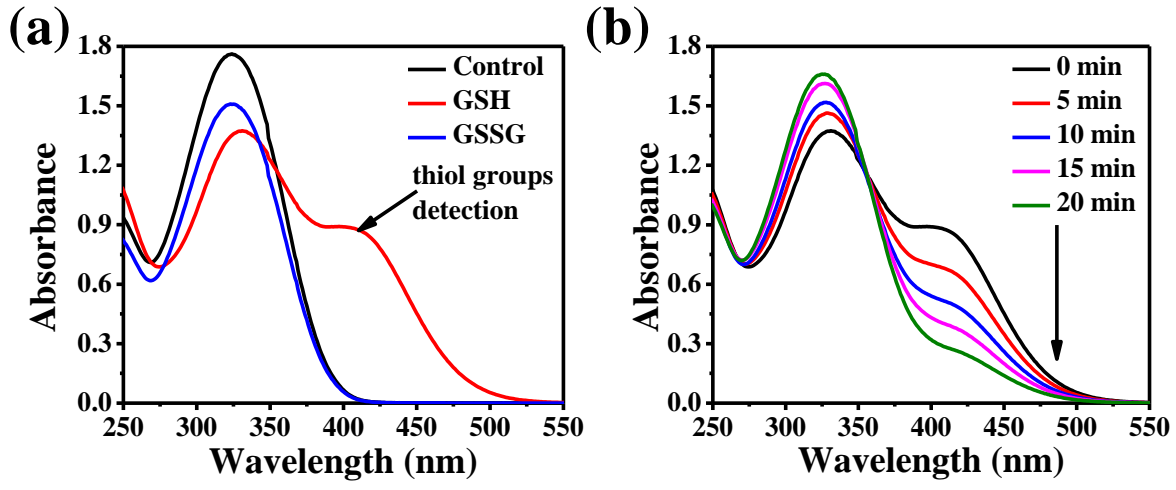

Figure S7. Ellman's reagent used for determination of thiol groups. (a) UV-vis absorption spectra of $100 \mu \mathrm{M}$ GSH and GSSG after reaction with colorless DTNB. The control means the UV-vis absorption of DNTB. (b) Evolution of UV-vis absorption spectra for GSH $(100 \mu \mathrm{M})$ in the presence $10 \mu \mathrm{g} / \mathrm{mL}$ GQD irradiated for selected times. 
(a)

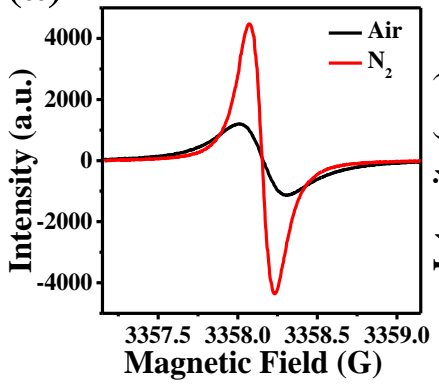

(b)

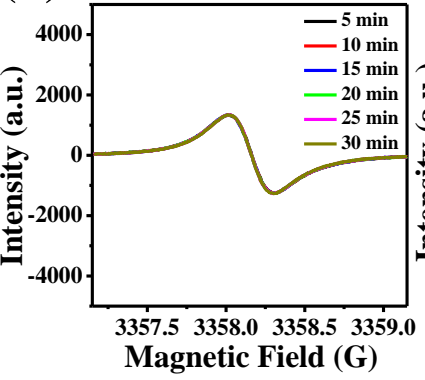

(c)

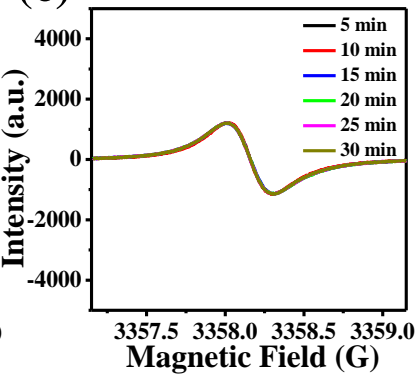

Figure S8. Effects of GQD on the lipid peroxidation in liposomes. (a) ESR spectra of ${ }^{15} \mathrm{~N}-\mathrm{PDT}$ in nitrogen purged or air-saturated aqueous solutions. (b) ESR spectra obtained at selected irradiation times for samples containing $30 \mathrm{mg} / \mathrm{mL}$ EYPC liposomes, $0.2 \mathrm{mM}{ }^{15} \mathrm{~N}$-PDT with $10 \mathrm{mM}$ PBS $(\mathrm{pH}=7.27)$. (c) ESR spectra obtained at selected irradiation times for samples containing $30 \mathrm{mg} / \mathrm{mL}$ EYPC liposomes, $0.2 \mathrm{mM}{ }^{15} \mathrm{~N}-\mathrm{PDT}$ and $100 \mu \mathrm{g} / \mathrm{mL}$ GQD. 